\title{
Isoforskolin downregulates proinflammatory responses induced by Borrelia burgdorferi basic membrane protein A
}

\author{
HUA ZHAO $^{1 *}$, AIHUA LIU ${ }^{2,3 *}$, LONGQIANG SHEN ${ }^{2}$, CUIPING XU $^{2}$, ZIWEI ZHU $^{1}$, \\ JIARU YANG ${ }^{2}$, XINLING HAN $^{1}$, FUKAI BAO ${ }^{1,3}$ and WEIMIN YANG ${ }^{4}$ \\ Departments of ${ }^{1}$ Microbiology and Immunology and ${ }^{2}$ Biochemistry and Molecular Biology; \\ ${ }^{3}$ Institute for Tropical Medicine; ${ }^{4}$ School of Pharmaceutical Science and Yunnan Key Laboratory of \\ Pharmacology for Natural Products, Kunming Medical University, Kunming, Yunnan 650500, P.R. China
}

Received December 21, 2016; Accepted August 10, 2017

DOI: $10.3892 / e t m .2017 .5300$

\begin{abstract}
The plant Coleus forskohlii is distributed primarily in India, Thailand, China, Egypt and Brazil and has a history of use in the treatment of multiple diseases. Isoforskolin (ISOF) is the principle active component of $C$. forskohlii native to China and has previously been studied for its biological effects. The aim of the present study was to evaluate the effect of ISOF on the proinflammatory responses induced by recombinant Borrelia burgdorferi basic membrane protein A (rBmpA). In in vitro experiments, the proinflammatory effects of $\mathrm{rBmpA}$ and the anti-inflammatory function of ISOF were evaluated in murine macrophages, human macrophages and dendritic cells by detecting the transcription and expression of tumor necrosis factor (TNF)- $\alpha$ and interleukin (IL)-6. In in vivo experiments, mean arthritis index and X-ray and histopathological examinations were used to verify the role of ISOF in experimental Lyme arthritis in mice. The results indicated that $\mathrm{rBmpA}$, which induced the transcription and expression of TNF- $\alpha$ and IL-6, activated proinflammatory responses in murine macrophages, human macrophages and dendritic cells. In turn, ISOF downregulated the transcription and expression of TNF- $\alpha$ and IL-6 induced by rBmpA. Additionally, the in vivo experiments demonstrated that ISOF could also inhibit the symptoms of
\end{abstract}

Correspondence to: Professor Fukai Bao, Department of Microbiology and Immunology, Kunming Medical University, 1186 Chunronxi Road, Kunming, Yunnan 650500, P.R. China

E-mail: baofukai@kmmu.edu.cn

Professor Weimin Yang, School of Pharmaceutical Science and Yunnan Key Laboratory of Pharmacology for Natural Products, Kunming Medical University, 1186 Chunrongxi Road, Kunming, Yunnan 650500, P.R. China

E-mail: ywmbessie@yeah.net

*Contributed equally

Key words: isoforskolin, Borrelia burgdorferi, basic membrane protein A, Lyme disease experimental Lyme arthritis. These results suggest that ISOF may have a potential application as an anti-inflammatory agent for the treatment of Lyme arthritis.

\section{Introduction}

Lyme disease, a widely epidemic tick-borne zoonosis, is caused by the spirochete Borrelia burgdorferi (B. burgdorferi) and is spread to humans through the bites of infected ticks (1). Areas of high risk include the USA, Northern Europe and parts of Eastern Asia (1). Arthritis, characterized by recurrent attacks or persistent swelling in certain large joints, typically develops during the middle or late stages of Lyme disease (2). In a number of patients with Lyme arthritis, the earlier clinical features of Lyme disease, including skin rash, headache and fever are not evident (2).

B. burgdorferi lacks toxin production and during infection, the majority of tissue damage results from host inflammatory reactions rather than from the pathogen itself (2). Cytokines, including tumor necrosis factor (TNF)- $\alpha$, interleukin (IL)-6 and IL-1 $\beta$ serve key functions in the pathogenesis of Lyme arthritis (3). In particular, these cytokines activate phagocytes in order to eliminate pathogens and attract other immune cells that aid in the host inflammatory response (3). In a previous study by our group, it was identified that the B. burgdorferi basic membrane protein $\mathrm{A}(\mathrm{BmpA})$ was involved in the pathogenesis of Lyme arthritis (4). Notably, the BmpA gene of Lyme-causing spirochete was preferentially upregulated in mouse joints compared with skin, heart and bladder tissues, and B. burgdorferi lacking BmpA failed to induce arthritis (4). It has also been reported that recombinant $\mathrm{BmpA}(\mathrm{rBmpA})$ may activate proinflammatory responses in human synovial cells and induce the expression of TNF- $\alpha$ and IL-1 $\beta$ (5).

The plant Coleus forskohlii (C. forskohlii) is primarily distributed in India, Thailand, China, Egypt and Brazil, and has a history of use in the treatment of multiple diseases, including heart disease, respiratory disorders, convulsion, intestinal disturbance and liver fatigue (6). Isoforskolin (ISOF) is the principle active component of $C$. forskohlii native to the southwest region of China, and has previously been studied for its anti-inflammatory effects $(7,8)$. Notably, a study performed by Yang et al (9) observed that ISOF 
reduced the secretion of lipopolysaccharide (LPS)-induced cytokines, namely TNF- $\alpha$, IL-1 $\beta$, IL-6 and IL-8, in human mononuclear leukocytes. The present study hypothesized that ISOF suppressed BmpA-induced inflammation. The results demonstrated the transcription and expression of TNF- $\alpha$ and IL- 6 in vitro and estimated the mean arthritis index (MAI), X-ray and histopathological examinations in in vivo experiments in mice.

\section{Materials and methods}

Reagents and rBmpA preparation. $\mathrm{rBmpA}$ was produced in Escherichia coli BL21 (GE Healthcare, Chicago, IL, USA) using the bacterial expression vector pGEX-6P1 (GE Healthcare) and the following primers with EcoRI and XhoI restriction sites: Forward, 5'-ACGAATTCATGAATAAAA TATTGTTGTTGA-3' and reverse, 5'-AGCTCGTAAATA AATTCTTTAAGAAA-3' (4,5). Pure ISOF was provided by Dr Weimin Yang [School of Pharmaceutical Science and Yunnan Key Laboratory of Pharmacology for Natural Products, Kunming Medical University (KMU), Kunming, China] and reconstituted at $1 \times 10^{5} \mu \mathrm{M} / \mathrm{ml}$ in dimethyl sulfoxide (DMSO) for stock solution. LPS and phorbol-12-myristate-13-acetate (PMA) were purchased from Sigma-Aldrich (Merck KGaA, Darmstadt, Germany) and ionomycin was purchased from Apollo Scientific Ltd. (Stockport, UK). Recombinant human (rh)IL-4, rhTNF- $\alpha$ and rh-granulocyte macrophage colony-stimulating factor (rhGM-CSF) were purchased from R\&D Systems, Inc. (Minneapolis, MN, USA).

Cell culture. The murine macrophage cell line RAW264.7 and human monocytic leukemia cell line THP-1 were obtained from the Kunming Institute of Zoology, Chinese Academy of Sciences (Kunming, China). All cell culture medium, serum and antibiotics were purchased from Gibco; Thermo Fisher Scientific, Inc. (Waltham, MA, USA). All cells were cultured at $37^{\circ} \mathrm{C}$ with a humidified atmosphere of $5 \% \mathrm{CO}_{2}$. Cellular supernatants were collected for ELISA. Cell lysates were prepared using RNAiso Plus reagent (Takara Bio, Inc., Otsu, Japan).

For RAW264.7 cells, Dulbecco's modified Eagle's medium (high glucose) supplemented with 10\% FBS and $1 \%$ penicillin-streptomycin was used as culture medium. RAW264.7 cells were seeded in 96-well microplates at a concentration of $1 \times 10^{5}$ cells $/ \mathrm{ml}$ and stimulated with $20 \mu \mathrm{g} / \mathrm{ml}$ $\mathrm{rBmpA}$ or $20 \mu \mathrm{g} / \mathrm{ml} \mathrm{rBmpA}+100 \mu \mathrm{mol} / \mathrm{ml}$ ISOF ( $\mathrm{n}=4$ wells per condition) for 24 and $48 \mathrm{~h}$. Additionally, a group of cells was cultured in $10 \% \mathrm{FBS} / \mathrm{DMEM}$ medium alone as a blank control and subgroups of these cells were stimulated with $0.1 \%$ DMSO (as a solvent control) or $1 \mu \mathrm{g} / \mathrm{ml}$ LPS (as a positive control).

For THP-1 cells, RPMI-1640 supplemented with $10 \%$ FBS and $1 \%$ penicillin-streptomycin was used as cell culture. THP-1 cells at $5 \times 10^{5}$ cells $/ \mathrm{ml}$ were differentiated into macrophages by $100 \mathrm{ng} / \mathrm{ml}$ PMA pretreatment for $24 \mathrm{~h}$ (10). THP-1 cells at $2 \times 10^{5}$ cells $/ \mathrm{ml}$ were pretreated with $200 \mathrm{ng} / \mathrm{ml} \mathrm{rhIL}-4$, $100 \mathrm{ng} / \mathrm{ml} \mathrm{rhGM-CSF}, 10 \mathrm{ng} / \mathrm{ml} \mathrm{rhTNF}-\alpha$ and $200 \mathrm{ng} / \mathrm{ml}$ ionomycin for 2 days for differentiation into mature dendritic cells (11). Subsequently, the macrophages and dendritic cells were cultured in 10\% FBS/RPMI-1640 and stimulated with
$20 \mu \mathrm{g} / \mathrm{ml} \mathrm{rBmpA}, 20 \mu \mathrm{g} / \mathrm{ml} \mathrm{rBmpA}+100 \mu \mathrm{mol} / \mathrm{ml}$ ISOF, $0.1 \%$ DMSO (solvent control) or $1 \mu \mathrm{g} / \mathrm{ml}$ LPS (positive control) ( $\mathrm{n}=4$ wells per condition) for 24, 48 and $72 \mathrm{~h}$. Additionally, a group of cells was maintained in RPMI 1640 supplemented with $10 \%$ FBS medium as a blank control.

ELISA. The concentrations of TNF- $\alpha$ and IL-6 in the cellular supernatant samples were measured using Mouse TNF- $\alpha$ ELISA kit (cat. no. DKW12-2720-096; Dakawe, Shenzhen, China), Mouse IL-6 ELISA kit (cat. no. DKW12-2720-096; Dakawe), Human TNF- $\alpha$ ELISA kit (cat. no. ELH-TNF $\alpha-001$; Ray Biotech, Inc., Norcross, GA, USA) and Human IL-6 ELISA kit (cat. no. ELH-IL6-001; Ray Biotech, Inc.) were used according to the manufacturers' protocols.

RNA extraction and reverse transcription-quantitative polymerase chain reaction (RT-qPCR). TNF- $\alpha$ and IL-6 mRNA expression was assessed by RT-qPCR. Total RNA was extracted from cells using the RNAiso Plus reagent, and a PrimeScript Reverse Transcription reagent kit with gDNA Eraser (Takara Bio, Inc.) was used to synthesize cDNA, according to the manufacturer's protocol. mRNA expression was detected using the standard SYBR-Green RT-PCR kit (Takara Bio, Inc.) according to the manufacturer's protocol. The sequences of the specific primers used to amplify murine and human TNF- $\alpha$, IL- 6 and $\beta$-actin were as follows: For murine TNF- $\alpha$, forward, 5'-GGTCCCCAAAGGGATGAG AA-3' and reverse, 5'-TGAGGGTCTGGGCCATAGAA-3'; for murine IL-6, forward, 5'-TCGGAGGCTTAATTACACATG TTC-3' and reverse, 5'-CATACAATCAGAATTGCCATT GC-3'; for murine $\beta$-actin, forward, 5'-TGCCGCATCCTCTTC CTC-3' and reverse, 5'-CGCCTTCACCGTTCCAGT-3'; for human TNF- $\alpha$, forward, 5'-CCTCTCTCTAATCAGCCCTCT G-3' and reverse, 5'-GAGGACCTGGGAGTAGATGAG-3'; for human IL-6, forward, 5'-ACTCACCTCTTCAGAACG AATTG-3' and reverse, 5'-CCATCTTTGGAAGGTTCAGGT TG-3'; and for human $\beta$-actin, forward, 5'-CAAGGCCAACCG CGAGAAGA-3' and reverse, 5'-GGATAGCACAGCCTGGAT AG-3' (12). qPCR was performed using SYBR Premix Ex Taq (Takara Bio, Inc.), according to the manufacturer's protocol. The reaction conditions were $95^{\circ} \mathrm{C}$ for $10 \mathrm{~min}$, and 40 cycles of denaturation at $95^{\circ} \mathrm{C}$ for $15 \mathrm{sec}$ and annealing/elongation at $60^{\circ} \mathrm{C}$ for $30 \mathrm{sec}$. The relative expression was analyzed by the $2^{-\Delta \Delta \mathrm{Cq}}$ method (12).

Animals. A total of 45 female Kunming mice (age, 6-8 weeks; weight, 18-22 g) were acquired from the Experimental Animal Center of KMU, Kunming, China. All animals were cared for according to the Guide for the Care and Use of Experimental Animals formulated by the Chinese Council on Animal Care and the Principles of Laboratory Animal Care formulated by Yunnan Provincial Council. The experimental protocol received approval from the Animal Use and Care Committee of KMU and was performed according to Public Health Service Policy on Humane Care and Use of Laboratory Animals by the National Institute of Health (13). The mice were kept in standardized units with filtered air at a controlled temperature of $18-22^{\circ} \mathrm{C}$ and humidity of 50-60\% with a $12 \mathrm{~h}$ light/dark cycle and free access to food and water. Prior to experimental procedures, the mice were adapted to the environment for 
1 week. The surgical procedures performed in animals were under general anesthesia using $360 \mathrm{mg} / \mathrm{kg}$ chloral hydrate [intraperitoneal (i.p.)].

Reagents. The preparation of ISOF stock and purified $\mathrm{rBmpA}$ was performed using the aforementioned methods. Phosphate-buffered saline (PBS) was purchased from Thermo Fisher Scientific, Inc., and diluted to $0.01 \mathrm{M}$ with ultrapure water. The $0.01 \mathrm{M}$ PBS was then used to dilute ISOF stock $\left(1 \times 10^{5} \mathrm{Mm} / \mathrm{ml}\right)$ and purified $\mathrm{rBmpA}$ to the concentration of $0.05 \mathrm{mg} / \mathrm{ml}$.

Grouping of mice and treatment protocol. Mice were randomly divided into a PBS control group $(n=15)$, Lyme arthritis group $(n=15)$ and ISOF group $(n=15)$. The Lyme arthritis model was established in the Lyme arthritis and ISOF groups as follows: $50 \mu 1 \mathrm{rBmpA}(0.05 \mathrm{mg} / \mathrm{ml})$ was injected into the tibiotarsal joint cavity of both hind legs once every 3 days for a total of four injections. PBS (0.01 M) or ISOF solution ( $1 \mathrm{mg} / \mathrm{kg}$ weight, i.p.) was then administered to the mice in the Lyme arthritis and ISOF groups, respectively. In the control group, 0.01 M PBS was administered instead of rBmpA and ISOF.

Evaluation of mice. To assess the extent of arthritis, two researchers blinded to the experimental conditions scored the tibiotarsal joints twice a week following the initial injection. The joints were evaluated according to the following arthritis scoring scale: 0 , no joint swelling or redness; 1 , slight reddening of the joint but no swelling; 2 , slight reddening and swelling of the joint; 3 , moderate reddening and swelling of the joint; 4, notable swelling and dysfunction of the joint. The MAI in each group was equal to the sum of arthritis scores divided by the number of mice hind legs. At 4 weeks after discontinuation of injections, dental radiography units were used to obtain X-ray images.

Histopathological examination. All 45 mice were anaesthetized with $360 \mathrm{mg} / \mathrm{kg}$ chloral hydrate (i.p.) and euthanized 4 weeks following the last injection in order to collect the bilateral knee joint, the tibiotarsal joint and the entire paw. The joint tissue was fixed in $4 \%$ paraformaldehyde at room temperature for $24 \mathrm{~h}$. Following decalcification with EDTA, all samples were cut into sections $5 \mu \mathrm{m}$ thick and stained using hematoxylin and eosin at room temperature. Stained sections were observed and photographed with a light inverted microscope.

Statistical analysis. Data were expressed as the mean \pm standard error of the mean. Differences between the mean values of the groups were evaluated by one-way analysis of variance followed by a Student-Newman-Keuls test using the statistical analysis software GraphPad Prism 6.0 (GraphPad Software, Inc., La Jolla, CA, USA). $\mathrm{P}<0.05$ was considered to indicate a statistically significant difference.

\section{Results}

TNF- $\alpha$ concentrations in cellular supernatants from murine macrophages, human macrophages and human dendritic
A

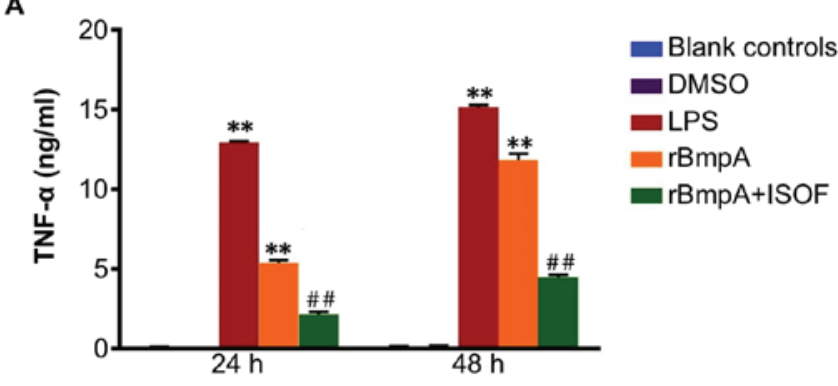

B

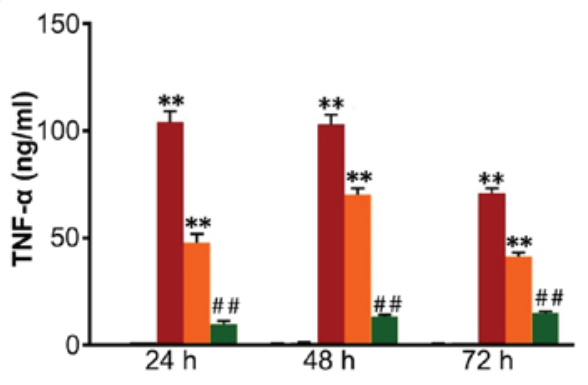

Blank controls

- DMSO

- LPS

- rBmpA

- rBmpA+ISOF

C

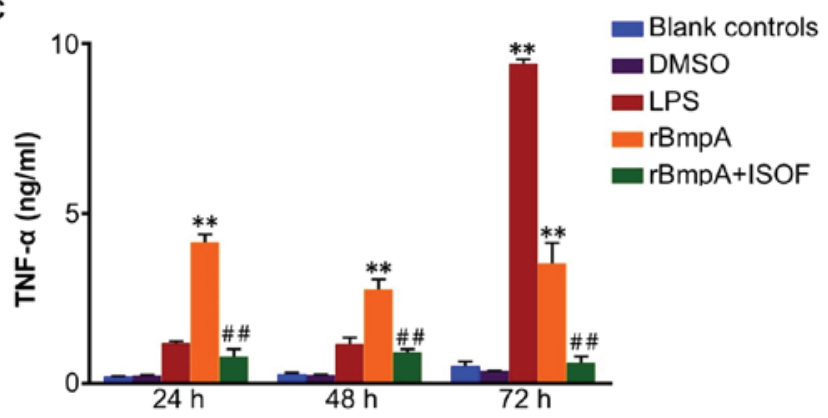

Figure 1. TNF- $\alpha$ concentrations in the cellular supernatants of murine macrophages, human macrophages and human dendritic cells with different stimulations. (A) TNF- $\alpha$ concentrations in murine macrophages. (B) TNF- $\alpha$ concentrations inhuman macrophages. (C) TNF- $\alpha$ concentrations inhuman dendritic cells. ${ }^{* *} \mathrm{P}<0.01$ vs. blank controls; ${ }^{\# \#} \mathrm{P}<0.01$ vs. rBmpA group. TNF- $\alpha$, tumor necrosis factor- $\alpha$; DMSO, dimethyl sulfoxide; LPS, lipopolysaccharide; rBmpA, recombinant Borrelia burgdorferi basic membrane protein A; ISOF, isoforskolin.

cells. The TNF- $\alpha$ concentrations in the cellular supernatants of the different cell groups were detected. TNF- $\alpha$ was quantified at different culture time points using specific ELISA kits. In the supernatant from murine macrophages at 24 and $48 \mathrm{~h}$, it was observed that treatment with LPS or rBmpA significantly increased the level of TNF- $\alpha$ when compared with the blank controls (all $\mathrm{P}<0.01$; Fig. 1A). In turn, ISOF treatment significantly reduced the secretion of TNF- $\alpha$ induced by $\mathrm{rBmpA}$ (all $\mathrm{P}<0.01$; Fig. 1A).

Similarly, LPS or rBmpA treatment induced a significant increase in TNF- $\alpha$ in the supernatant of human macrophages at 24, 48 and $72 \mathrm{~h}$ compared with the blank control group (all $\mathrm{P}<0.01$; Fig. 1B). Additionally, treatment with ISOF significantly reduced the secretion of TNF- $\alpha$ induced by $\mathrm{rBmpA}$ at 24,48 and $72 \mathrm{~h}$ compared with the rBmpA group (all $\mathrm{P}<0.01$; Fig. 1B).

Furthermore, as depicted in Fig. 1C, rBmpA upregulated the levels of TNF- $\alpha$ in the supernatants of human dendritic cells after 24, 48 and $72 \mathrm{~h}$, and LPS after $72 \mathrm{~h}$ compared with the blank controls (all $\mathrm{P}<0.01$ ). Meanwhile, ISOF treatment 
A
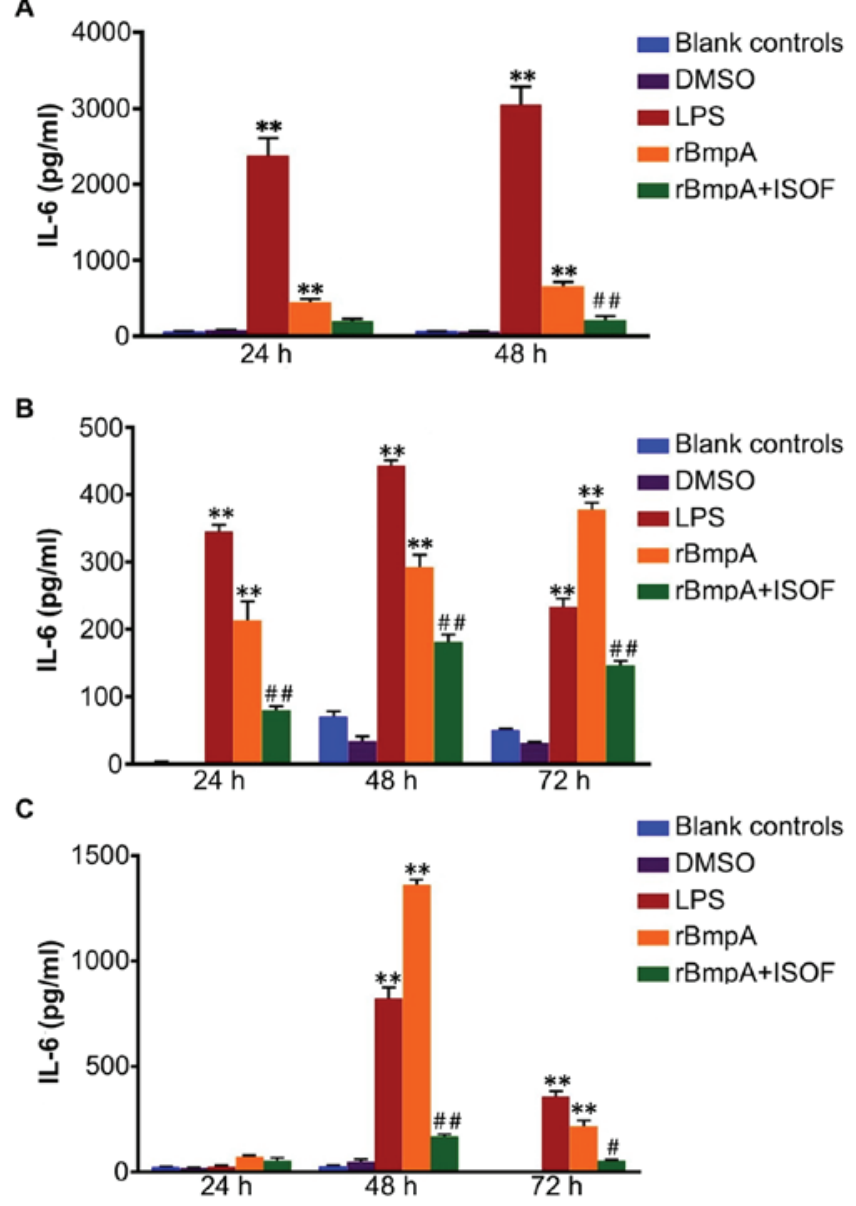

Figure 2. IL-6 concentrations in the cellular supernatants of murine macrophages, human macrophages and human dendritic cells with different stimulations. (A) IL-6 concentrations in murine macrophages. (B) IL-6 concentrations in human macrophages. (C) IL-6 concentrations in human dendritic cells. ${ }^{* *} \mathrm{P}<0.01$ vs. blank controls; ${ }^{~} \mathrm{P}<0.05$ vs. rBmpA group; ${ }^{\# \#} \mathrm{P}<0.01$ vs. rBmpA group. IL-6, interleukin-6; DMSO, dimethyl sulfoxide; LPS, lipopolysaccharide; rBmpA, recombinant Borrelia burgdorferi basic membrane protein A; ISOF, isoforskolin.

significantly suppressed the rBmpA-induced secretion of TNF- $\alpha$ at 24,48 and $72 \mathrm{~h}(\mathrm{P}<0.01)$.

IL-6 concentrations in cellular supernatants from murine macrophages, human macrophages and human dendritic cells. As for TNF- $\alpha$, ELISA was used to quantify the supernatant concentrations of IL- 6 at different culture time points. For murine macrophages, compared with the blank control group, the LPS or rBmpA groups exhibited significantly increased supernatant levels of IL-6 at 24 and 48 h ( $<<0.01$; Fig. 2A); In turn, ISOF treatment reduced the secretion of IL- 6 induced by $\mathrm{rBmpA}$, which was deemed to be significant at $48 \mathrm{~h}(\mathrm{P}<0.01)$ but not at $24 \mathrm{~h}$ (Fig. 2A).

Accordingly, IL-6 levels were significantly increased in the supernatants of human macrophages by LPS or rBmpA compared with the blank controls at 24, 48 and $72 \mathrm{~h}(\mathrm{P}<0.01)$. Meanwhile, ISOF treatment significantly reduced the secretion of IL- 6 induced by rBmpA at 24,48 and $72 \mathrm{~h}(\mathrm{P}<0.01$; Fig. 2B).

For human dendritic cells, the supernatant levels of IL-6were significantly increased by the LPS and
A

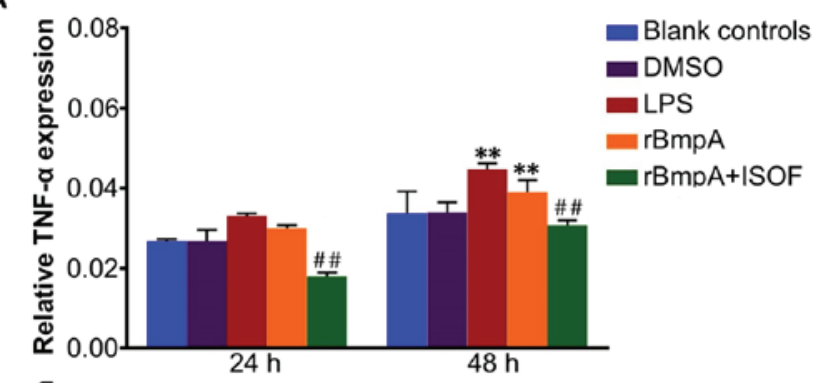

B

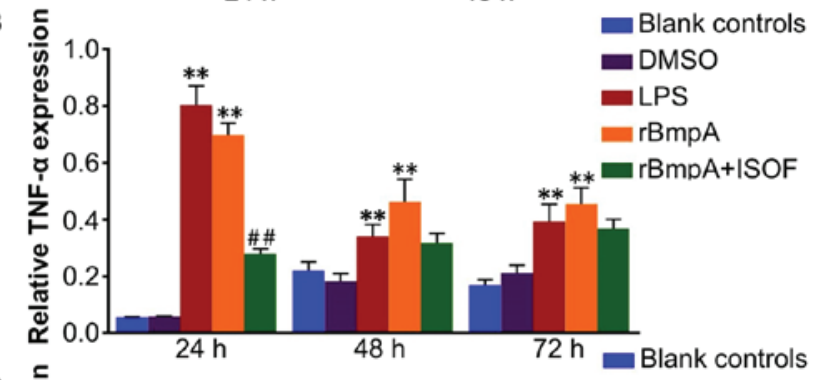

C

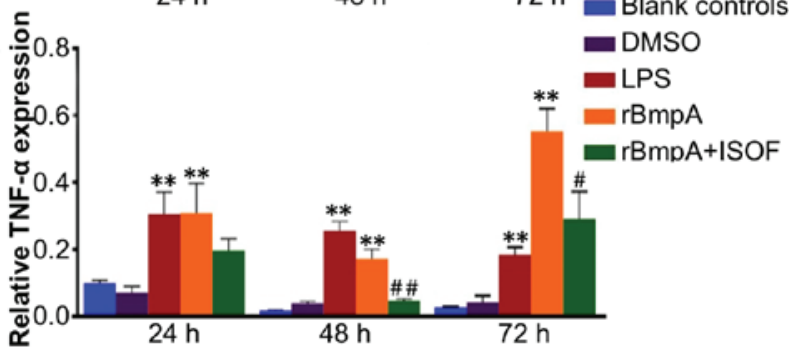

Figure 3. Relative TNF- $\alpha$ mRNA expression in murine macrophages, human macrophages and human dendritic cells with different stimulations. (A) TNF- $\alpha$ mRNA expression in murine macrophages. (B) TNF- $\alpha$ mRNA expression in human macrophages. (C) TNF- $\alpha$ mRNA expression in human dendritic cells. ${ }^{* *} \mathrm{P}<0.01$ vs. blank controls; ${ }^{*} \mathrm{P}<0.05$ vs. $\mathrm{rBmpA}$ group; ${ }^{\# \#} \mathrm{P}<0.01$ vs. rBmpA group. TNF- $\alpha$, tumor necrosis factor- $\alpha$; DMSO, dimethyl sulfoxide; LPS, lipopolysaccharide; rBmpA, recombinant Borrelia burgdorferi basic membrane protein A; ISOF, isoforskolin.

rBmpA at 48 and $72 \mathrm{~h}$, compared with the blank controls $(\mathrm{P}<0.01)$. Treatment with ISOF significantly reduced the rBmpA-induced increase in IL-6 at 48 and $72 \mathrm{~h}(\mathrm{P}<0.01$ and $\mathrm{P}<0.05$, respectively; Fig. $2 \mathrm{C}$ ).

TNF- $\alpha$ mRNA levels in murine macrophages, human macrophages and human dendritic cells. TNF- $\alpha$ mRNA levels in murine macrophages, human macrophages and human dendritic cells were determined by RT-qPCR. Murine TNF- $\alpha$ expression was significantly increased by LPS or rBmpA at $48 \mathrm{~h}$ compared with the blank control group $(\mathrm{P}<0.01$; Fig. 3A). ISOF downregulates murine TNF- $\alpha$ expression induced by the rBmpA at 24 and $48 \mathrm{~h}(\mathrm{P}<0.01)$. For human macrophages and human dendritic cells, the expression levels of TNF- $\alpha$ were significantly elevated in the LPS and $\mathrm{rBmpA}$ groups compared with the blank controls at 24, 48 and $72 \mathrm{~h}$ (all $\mathrm{P}<0.01$; Fig. 3B and $\mathrm{C}$ ). While ISOF treatment significantly reduced the $\mathrm{rBmpA}$-induced upregulation of TNF- $\alpha$ in in human macrophages at $24 \mathrm{~h}(\mathrm{P}<0.01$; Fig. 3B), and in human dendritic cells at 48 and $72 \mathrm{~h}(\mathrm{P}<0.01$ and $\mathrm{P}<0.05$, respectively).

IL-6 mRNA expression levels in murine macrophages, human macrophages and human dendritic cells. The IL-6 
A

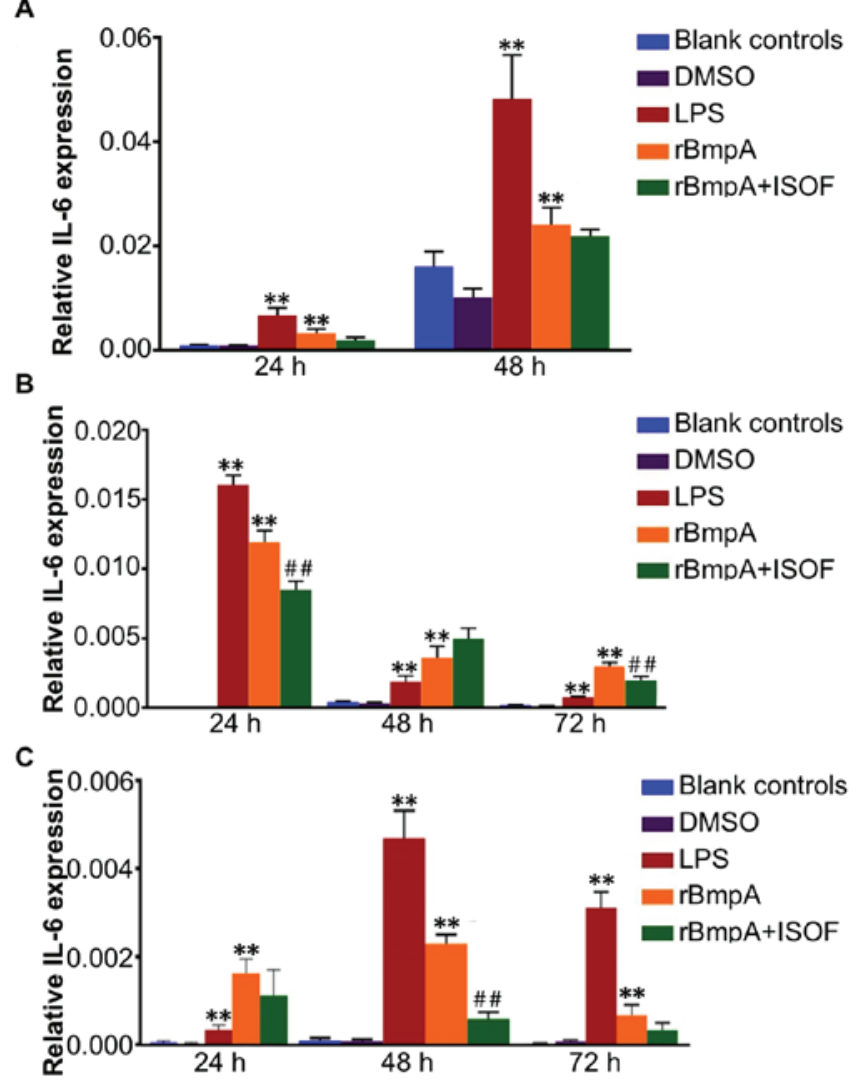

Figure 4. Relative IL-6 mRNA expression in murine macrophages, human macrophages and human dendritic cells with different stimulations. (A) IL-6 mRNA expression in murine macrophages. (B) IL-6mRNA expression in human macrophages. (C) Il-6 mRNA expression in human dendritic cells. ${ }^{* *} \mathrm{P}<0.01$ vs. blank controls; ${ }^{\# \#} \mathrm{P}<0.01$ vs. rBmpA group. IL-6, interleukin-6; DMSO, dimethyl sulfoxide; LPS, lipopolysaccharide; rBmpA, recombinant Borrelia burgdorferi basic membrane protein A; ISOF, isoforskolin.

mRNA levels in murine macrophages, human macrophages and human dendritic cells were determined by RT-qPCR. The results indicated that IL- 6 expression levels in murine macrophages, human macrophages and human dendritic cells were significantly increased by LPS or $\mathrm{rBmpA}$ treatment when compared with the blank controls at all time points (all $\mathrm{P}<0.01$; Fig. 4). While ISOF treatment reduced the expression of IL- 6 induced by rBmpA in human macrophages at 24 and $72 \mathrm{~h}$ (both $\mathrm{P}<0.01$; Fig. 4B) but not at 48 h. ISOF treatment also significantly decreased the mRNA expression of IL- 6 in rBmpA-stimulated human dendritic cells, which was deemed to be significant at $48 \mathrm{~h}(\mathrm{P}<0.01)$ but not at 24 or $72 \mathrm{~h}$ (Fig. $4 \mathrm{C})$.

ISOF effects in the mouse model of Lyme arthritis in vivo. On initiation of animal experimentation, mice in the control and ISOF groups exhibited similar feeding behavior and activity levels. At 1-2 days following the initial injection, the mice in the ISOF group presented with slight redness and swelling at the injection site, though this diminished after 3-4 days. The groups administered with rBmpA (Lyme arthritis group and ISOF group) presented slight redness and swelling in the tibiotarsal joints at day 4 after receiving the initial $\mathrm{rBmpA}$ injection. The symptoms peaked at days 14-18 and gradually resolved thereafter. A total of 3 mice in $\mathrm{rBmpA}$ group with

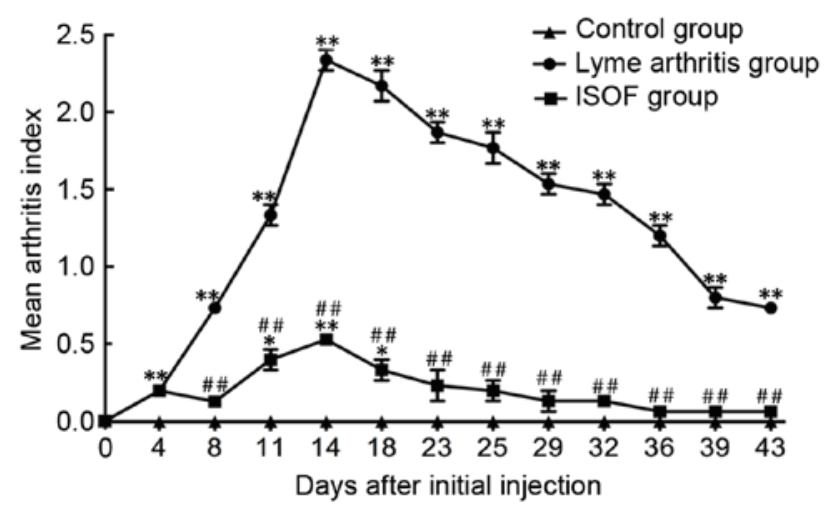

Figure 5. Mean arthritis index in mice with Lyme arthritis. ${ }^{* *} \mathrm{P}<0.01$ vs. control group; ${ }^{\#} \mathrm{P}<0.05$ vs. Lyme arthritis group. ISOF, isoforskolin.

severe inflammation exhibited deformities in the tibiotarsal joint and limited bending ability. The injection of $\mathrm{rBmpA}$ caused a significant increase in the MAI compared to that in the control group (all $\mathrm{P}<0.01$; Fig. 5). The treatment of ISOF significantly decreased the MAI compared to $\mathrm{rBmpA}$ group (all $\mathrm{P}<0.01$; Fig. 5).

In X-ray images (Fig. 6), the mice with experimental arthritis exhibited swelling of the soft tissue and joint, joint deformity, stiffness and narrow joint spaces. Meanwhile, the mice administered with ISOF exhibited no stiffness and less swelling in soft tissue.

On histopathological examination of control group tissues (Fig. 6G), a regular joint space and orderly arrangement of cells in the synovial membrane were observed. Additionally, the articular synovial surface was neat and there was no infiltration of inflammatory cells. For the arthritis group (Fig. 6H), the joint tissue sections exhibited narrowing of the joint space, increased synovial cells, thickened synovial tissues and obvious fibrous tissue hyperplasia when compared with the control tissues. Furthermore, a high number of inflammatory cells (including polymorphonuclear leukocytes, monocytes-macrophages and lymphocytes) had infiltrated and the synovial membrane and pannus had invaded the joint, causing joint structural damage between bones. For the ISOF group (Fig. 6I), the joint tissue sections exhibited normalization of the joint space and neater rows of joint synovial cells compared with the arthritis group tissues. While the synovial cells remained slightly thickened compared with control tissues, the articular surface appeared neat and no notable inflammatory cell infiltration was observed.

\section{Discussion}

B. burgdorferi infection in joints has been correlated with Lyme arthritis (2). In infected joints, recognition by the innate immune system is key to the host response (14). Bacterial lipoproteins are recognized by toll-like receptor 2 (TLR2), which induces macrophages and dendritic cells to secrete cytokines (14). By interacting with co-receptors, TLR2 represents a key recognition receptor on immune cells (3). Following immune recognition, the phagocytosis of $B$. burgdorferi by macrophages results in increased transcription of IL- $1 \beta$, IL-6 and TNF- $\alpha$, which activates dendritic cells by inducing the 


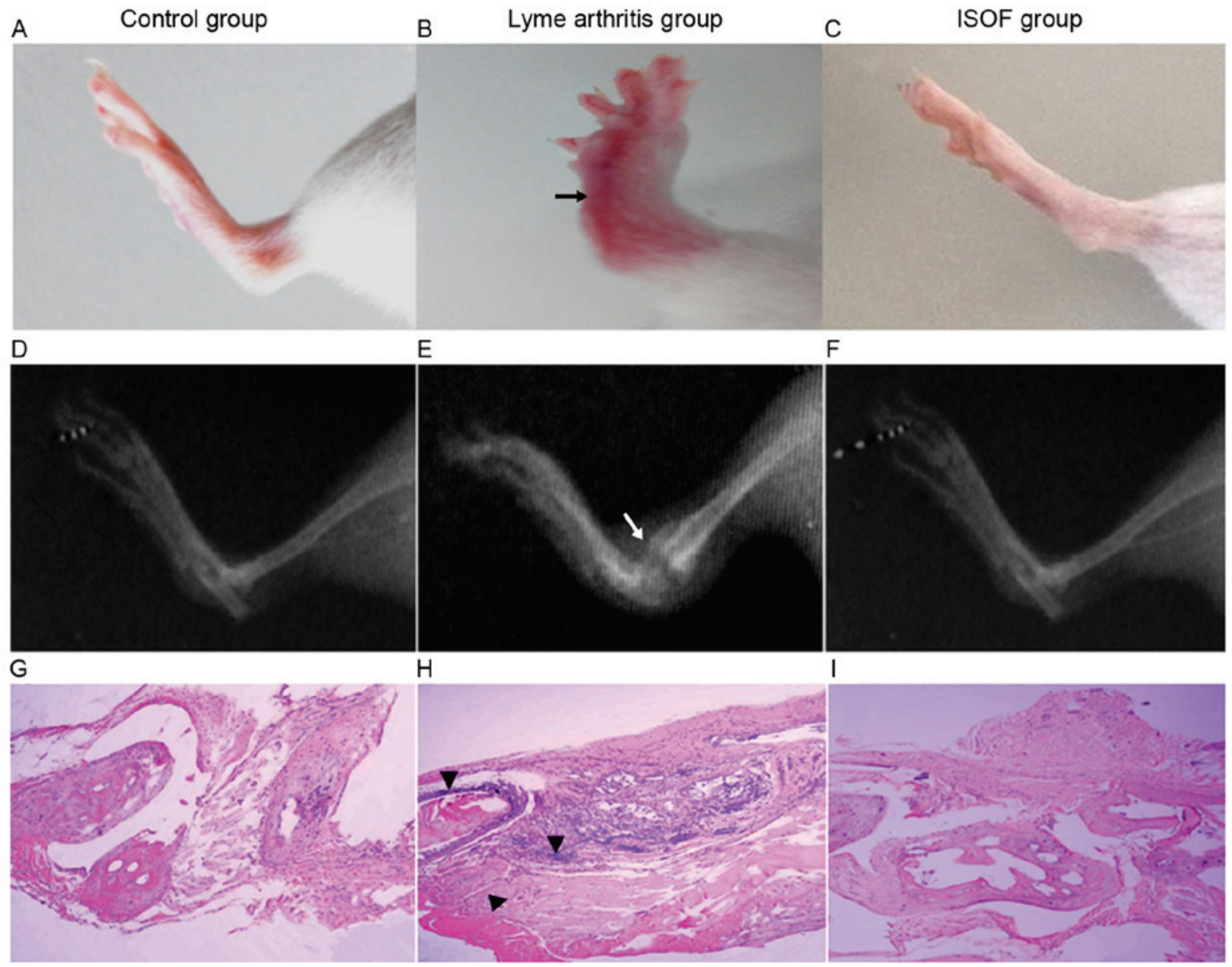

Figure 6. Comparison of the hind limbs of mice in each group at 4 weeks after the last injection. (A-C) Photographs of the mice hind limbs. Mice in Lyme arthritis group exhibited redness and swelling of the soft tissue and joint. (D-F) X-ray images. Swelling of the soft tissue and bone deformities were exhibited in Lyme arthritis group. (G-I) Light microscopic features in each group. More severe inflammation was observed in Lyme arthritis group (hematoxylin and eosin staining; magnification, x100). Arrows indicate pathological changes. ISOF, isoforskolin.

expression of CD83, and leads to increased transcription of IL-1 $\beta$, IL- 6 and TNF- $\alpha$ in dendritic cells (15).

In contrast to other gram-negative bacteria, B. burgdorferi does not express LPS, as the relevant genes are absent (3). The organism responds to different environments through specific gene expression and by altering the composition of its membrane proteins, which are involved in the pathogenesis of Lyme arthritis (16). The pathogen expresses numerous proteins on its outer surface, which are able to modulate the immune system of the host (3). BmpA is an immuno-dominant protein of $B$. burgdorferi as well as an arthritogenic factor $(4,17)$. BmpA activates proinflammatory responses in human synovial cells and induces the expression of TNF- $\alpha$ and IL-1 $\beta$ (5). The current study implicated $\mathrm{rBmpA}$ as a key activating factor of proinflammatory responses in murine macrophages, human macrophages and dendritic cells through the transcription and expression of TNF- $\alpha$ and IL-6.

The present study builds on previous findings regarding the role of ISOF in downregulating inflammatory responses. In animal models of LPS-induced acute lung injury, ISOF increased cyclic adenosine monophosphate levels and activated adenylyl cyclase isoforms 1, 2 and 5 (9). ISOF was also demonstrated to suppress LPS-induced secretion of cytokines, including TNF- $\alpha$, IL-1 $\beta$, IL-6 and IL- 8 , in human mononuclear leukocytes (9). LPS, as a component of the outer membrane of gram-negative bacteria, induces the activation of macrophages and dendritic cells, which leads to the release of cytokines, including TNF- $\alpha$, IL-1 $\beta$ and IL-6 $(18,19)$. The present study demonstrated that ISOF exerted general downregulatory effects on the transcription and expression of TNF- $\alpha$ and IL-6 induced by rBmpA in murine macrophages, human macrophages and human dendritic cells, and suppressed the inflammatory response and $\mathrm{rBmpA}$-induced arthritis in a mice model.

In conclusion, the present study indicated that $\mathrm{rBmpA}$, which induced the transcription and expression of TNF- $\alpha$ and IL-6, may activate proinflammatory responses in murine macrophages, human macrophages and dendritic cells and cause Lyme arthritis in mice. ISOF downregulated the transcription and expression of TNF- $\alpha$ and IL- 6 induced by rBmpA, and therefore may inhibit Lyme arthritis induced by rBmpA. Thus, ISOF may have a potential clinical application in the treatment of Lyme arthritis.

\section{Acknowledgements}

The present study was supported by grants from the National Natural Science Foundation of China (grant nos. 31560051, 
81560596 and 81173110) and the Natural Foundation of Yunnan Province (grant nos. 2012FB011, 2014FA011, 2014FB001 and 2014BC012).

\section{References}

1. Gerstenblith TA and Stern TA: Lyme disease: A review of its epidemiology, evaluation, and treatment. Psychosomatics 55: 421-429, 2014.

2. Stanek G, Wormser GP, Gray J and Strle F: Lyme borreliosis. Lancet 379: 461-473, 2012.

3. Oosting M, Buffen K, van der Meer JW, Netea MG and Joosten LA: Innate immunity networks during infection with Borrelia burgdorferi. Crit Rev Microbiol 42: 233-244, 2016.

4. Pal U, Wang P, Bao F, Yang X, Samanta S, Schoen R, Wormser GP, Schwartz I and Fikrig E: Borrelia burgdorferi basic membrane proteins A and B participate in the genesis of Lyme arthritis. J Exp Med 205: 133-141, 2008.

5. Yang X, Izadi H, Coleman AS, Wang P, Ma Y, Fikrig E, Anguita J and $\mathrm{Pal} \mathrm{U}$ : Borrelia burgdorferi lipoprotein BmpA activates proinflammatory responses in human synovial cells through a protein way. Microbes Infect 10: 1300-1308, 2008.

6. Yin J, Wang Y, Tan B, Kang Y, Xie D, Tian L and Huang J: Matrix solid-phase dispersion extraction for chromatographic analysis of labdane diterpenoids in Coleus forskohlii. Phytochem Anal 24: 117-123, 2013.

7. Tian L, Wang Y, Ling Y, Yin J, Chen J and Huang J: A sensitive and specific HPLC-MS/MS analysis and preliminary pharmacokinetic characterization of isoforskolin in beagle dogs J Chromatogr B Analyt Technol Biomed Life Sci 879: 3688-3693, 2011.

8. Feng S, Wang L, P. Voravuthikunchai S, Liang Z, Liu AH and Bao FK: Isoforskolin from native plant Coleus forskohlii of Yunnan, China plays multiple biological roles. Open J Immunol 6 : 63-66, 2016
9. Yang WM, Qiang DQ, Zhang M, Ma L, Zhang Y, Qing C, Xu Y, Zhen C, Liu J and Chen YH: Isoforskolin pretreatment attenuates lipopolysaccharide-induced acute lung injury in animal models. Int Immunopharmacol 11: 683-692, 2011.

10. Ringseis R, Schulz N, Saal D and Eder K: Troglitazone but not conjugated linoleic acid reduces gene expression and activity of matrix-metalloproteinases-2 and -9 in PMA-differentiated THP-1 macrophages. J Nutr Biochem 19: 594-603, 2008.

11. Berges C, Naujokat C, Tinapp S, Wieczorek H, Höh A, Sadeghi M, Opelz $\mathrm{G}$ and Daniel V: A cell line model for the differentiation of human dendritic cells. Biochem Bioph Res Comm 333: 896-907, 2005.

12. Livak KJ and Schmittgen TD: Analysis of relative gene expression data using real-time quantitative PCR and the 2(-Delta Delta C(T)) method. Methods 25: 402-408, 2001.

13. U.S. Department of Health And Human Services, National Institutes of Health, Office of Laboratory Animal Welfare: Public Health Service Policy on Humane Care and Use of Laboratory Animals, Revised 2015, NIH Publication No. 15-8013. Office of Laboratory Animal Welfare, National Institutes of Health, U.S. Department of Health and Human Services, Bethesda, MD, USA.

14. Nowalk AJ, Gilmore RD Jr and Carroll JA: Serologic proteome analysis of Borrelia burgdorferi membrane-associated proteins. Infect Immun 74: 3864-3873, 2006.

15. Bryksin AV, Tomova A, Godfrey HP and Cabello FC: BmpA is a surface-exposed outer membrane protein of Borrelia burgdorferi. FEMS Microbiol Lett 309: 77-83, 2010.

16. Hu L: Lyme Arthritis. Infect Dis Clin N Am 19: 947-961, 2005.

17. Cervantes JL, Hawley KL, Benjamin SJ, Weinerman B, Luu SM and Salazar JC: Phagosomal TLR signaling upon Borrelia burgdorferi infection. Front Cell Infect Microbiol 4: 55, 2014.

18. Zanoni I and Granucci F: Differences in lipopolysaccharide-induced signaling between conventional dendritic cells and macrophages. Immunobiology 215: 709-712, 2010.

19. Chow JC, Young DW, Golenbock DT, Christ WJ and Gusovsky F: Toll-like receptor-4 mediates lipopolysaccharide-induced signal transduction. J Biol Chem 274: 10689-10692, 1999. 